\title{
COMPLEX SUBMANIFOLDS OF CERTAIN NON-KAEHLER MANIFOLDS
}

\author{
By MAKoTO KIMURA
}

\section{$\S 0$. Introduction.}

Complex submanifolds of Kaehlerian manifolds have been studied extensively by many differential geometers (see, for example, the bibliography of Ogiue's paper [5]), but complex submanifolds of non-Kaehlerian Hermitian manifold have not been explored to any great extent.

On the other hand, E. Calabi and B. Eckmann [2] proved that the product of two odd-dimensional spheres which admits a Hermitian structure is called Calabi-Eckmann manifold. A Calabi-Eckmann manifold has two structures, namely the product Riemannian structure and the complex structure that is mentioned above. Thus submanifolds of Calabi-Eckmann manifold have twosided property. One is that they are submanifolds of a product manifold and another is that they are submanifolds of a complex manifold.

In $\S 1$, we study first of all, submanifolds of Riemannian product manifolds using the same method by G.D. Ludden and M. Okumura [3].

In $\S 2$, we study properties of complex submanifolds of a Riemannian product of two Sasakian manifolds and prove that any compact complex submanifold of certain non-Kaehlerian, Hermitian manifold, which is a generalization of CalabiEckmann manifold is minimal.

The author would like to express his hearty gratitude to Professor M. Okumura for his valuable suggestions, and he wishes to express his deep gratitude to Professor S. Tanno who took care for the publication of this paper on KODAI MATHEMATICAL JOURNAL.

\section{$\S 1$. Submanifold of Riemannian product manifolds.}

Let $\bar{M}_{1}, \bar{M}_{2}$ be respectively differentiable manifolds of dimensions $n$ and $m$, and we consider the product manifold $\bar{M}_{1} \times \bar{M}_{2}$. We denote by $\bar{P}_{\imath}(i=1,2)$ the projection mappings of the tangent space of $\bar{M}_{1} \times \bar{M}_{2}$ to that of $\bar{M}_{2}(i=1,2)$, where the tangent space to $\bar{M}_{1}$ (resp. $\bar{M}_{2}$ ) is identified with that of $\bar{M}_{1} \times$ (point) (resp. (point) $\times \bar{M}_{2}$ ). Then we have

$$
\bar{P}_{1}+\bar{P}_{2}=I, \quad \bar{P}_{1}^{2}=\bar{P}_{1}, \quad \bar{P}_{2}^{2}=\bar{P}_{2}, \quad \bar{P}_{1} \bar{P}_{2}=\bar{P}_{2} \bar{P}_{1}=0,
$$

Received March 28, 1984 
where $I$ denotes the identity transformation of the tangent space of $\bar{M}_{1} \times \bar{M}_{2}$. We put $\bar{P}=\bar{P}_{1}-\bar{P}_{2}$. Then it follows that

$$
\bar{P}^{2}=I, \quad \operatorname{tr} \bar{P}=m-n,
$$

where $\operatorname{tr} \bar{P}$ denotes the trace $\bar{P}$. We call $\bar{P}$ an almost product structure on $\bar{M}_{1} \times \bar{M}_{2}$ (cf. [3]).

If the manifolds $\bar{M}_{1}, \bar{M}_{2}$ are both Riemannian manifolds, we define a Riemannian metric of $\bar{M}_{1} \times \bar{M}_{2}$ by

$$
\bar{g}(X, Y)=\bar{g}_{1}\left(\bar{P}_{1} X, \bar{P}_{1} Y\right)+\bar{g}_{2}\left(\bar{P}_{2} X, \bar{P}_{2} Y\right),
$$

where $\bar{g}_{1}$ and $\bar{g}_{2}$ are respectively the Riemannian metrics of $\bar{M}_{1}$ and $\bar{M}_{2}$. Then it follows that

$$
\bar{g}(\bar{P} X, Y)=\bar{g}(X, \bar{P} Y), \quad \bar{\nabla}_{X} \bar{P}=0,
$$

where $\bar{\nabla}$ denotes the operator of covariant differentiation with respect to the Riemannian connection of $\bar{g}$.

Let $M^{n}$ be a submanifold of codimension $p$ in $\bar{M}_{1}^{m} \times \bar{M}_{2}^{n+p-m}$ and suppose $\imath: M \rightarrow \bar{M}_{1} \times \bar{M}_{2}$ the immersion. For a tangent vector field $X$ to $M$ and orthonormal normal vectors $N_{\alpha}(\alpha=1, \cdots, p)$ to $M$, the transforms $\bar{P} i_{*} X$ and $\bar{P} N_{\alpha}$ $(\alpha=1, \cdots, p)$ by $\bar{P}$ can be written as follows ;

$$
\begin{gathered}
\bar{P} i_{*} X=i_{*} P X+\sum_{\alpha=1}^{p} u^{\alpha}(X) N_{\alpha}, \\
\bar{P} N_{\alpha}=i_{*} U_{\alpha}+\sum_{\beta=1}^{p} \lambda_{\alpha \beta} N_{\beta} \quad(\beta=1, \cdots, p),
\end{gathered}
$$

where $P$ defines a symmetric linear transformation of the tangent bundle $T(M)$ of $M$, while $u^{\alpha}, U_{\alpha}$ and $\lambda_{\alpha \beta}$ define 1-forms, vector fields and functions on a neighborhood of a point of $M$ respectively. Moreover, we easily see that $g\left(U_{\alpha}, X\right)=u^{\alpha}(X)$, where $g$ is the induced Riemannian metric on $M$.

We denote by $\nabla$ the operator of covariant differentiation with respect to the Riemannian connection of $g$. Then Gauss and Weingarten equations are given by

$$
\begin{aligned}
\bar{\nabla}_{i * X} \imath_{*} Y & =i_{*} \nabla_{X} Y+\sigma(X, Y) \\
& =i_{*} \nabla_{X} Y+\sum_{\alpha=1}^{p} g\left(A_{N_{\alpha}} X, Y\right) N_{\alpha}, \\
\bar{\nabla}_{i * X} N_{\alpha} & =-\imath_{*} A_{N_{\alpha}} X+\nabla_{X}^{1} N_{\alpha}, \\
& =-\imath_{*} A_{N_{\alpha}} X+\sum_{\beta=1}^{p} s_{\alpha \beta}(X) N_{\beta},
\end{aligned}
$$

where $\sigma$ and $A$ are respectively the second fundamental form and the corresponding second fundamental tensor, while $\nabla^{\perp}, s_{\alpha \beta}$ the normal connection and the third fundamental tensor respectively. They satisfy

$$
\sigma(X, Y)=\sigma(Y, X), \quad s_{\alpha \beta}(X)=-s_{\beta \alpha}(X) .
$$




\section{$\S 2$. Complex submanifold of certain non-Kaehler manifolds.}

Let $\bar{M}=\bar{M}^{2 n+1}(\bar{\phi}, \bar{\xi}, \bar{\eta}, \bar{g})$ be a Sasakian manifold of dimension $2 n+1$. The structure tensors $(\bar{\phi}, \bar{\xi}, \bar{\eta}, \bar{g}$ ) satisfy (cf. [1])

$$
\begin{aligned}
& \bar{\phi}=-I+\bar{\eta} \otimes \bar{\xi}, \quad \bar{\phi} \bar{\xi}=0, \quad \bar{\eta}(\bar{\phi} X)=0, \quad \bar{\eta}(\bar{\xi})=1, \\
& \bar{g}(\bar{\phi} X, \bar{\phi} Y)=\bar{g}(X, Y)-\bar{\eta}(X) \bar{\eta}(Y), \quad \bar{\eta}(X)=\bar{g}(X, \bar{\xi}), \\
& \bar{\nabla}_{X} \bar{\xi}=\bar{\phi} X, \quad\left(\bar{\nabla}_{X} \bar{\phi}\right) Y=\bar{\eta}(Y) X-\bar{g}(X, Y) \bar{\xi} .
\end{aligned}
$$

Let $\bar{M}_{1}^{2 m+1} \times \bar{M}_{2}^{2 n+2 p-2 m-1}$ be the Riemannian product manifold of Sasakian manifolds, and let $\Sigma_{1}=\left(\bar{\phi}_{1}^{\prime}, \bar{\eta}_{1}^{\prime}, \bar{\xi}_{1}^{\prime}, \bar{g}_{1}^{\prime}\right)$ (resp. $\left.\Sigma_{2}=\left(\bar{\phi}_{2}^{\prime}, \bar{\eta}_{2}^{\prime}, \bar{\xi}_{2}^{\prime}, \bar{g}_{2}^{\prime}\right)\right)$ be the Sasakian structure on $\bar{M}_{1}$ (resp. $\left.\bar{M}_{2}\right)$. Then actions of $\bar{\phi}_{2}(i=1,2)$ on $\bar{M}_{1} \times \bar{M}_{2}$ are defined by $\bar{\phi}_{\imath}=\bar{\phi}_{i}^{\prime} \bar{P}_{2}$ where $\bar{P}_{1}$ (resp. $\bar{P}_{2}$ ) is the projection of tangent space of $\bar{M}_{1} \times \bar{M}_{2}$ to that of $\bar{M}_{1}$ (resp. $\bar{M}_{2}$ ), where the tangent space to $\bar{M}_{1}$ (resp. $\bar{M}_{2}$ ) is identified with that of $\bar{M}_{1} \times$ (point) (resp. (point) $\times \bar{M}_{2}$ ). Similarly, $\bar{\xi}_{\imath}$ and $\bar{\eta}_{\imath}$ are defined by $\bar{P}_{i} \bar{\xi}_{\imath}=\bar{\xi}_{\imath}^{\prime}, \bar{P}_{i} \bar{\xi}_{j}=0(i, j=1,2, i \neq j)$ and $\bar{\eta}_{i}(X)=\bar{\eta}_{i}^{\prime}\left(\bar{P}_{\imath} X\right)$ on $\bar{M}_{1} \times \bar{M}_{2}$.

For any tangent vector $X$ of $\bar{M}_{1} \times \bar{M}_{2}$, we define

$$
J X=\bar{\phi}_{1} X-\bar{\eta}_{2}(X) \bar{\xi}_{1}+\bar{\phi}_{2} X+\bar{\eta}_{1}(X) \bar{\xi}_{2} .
$$

Then $J$ defines a complex structure on $\bar{M}_{1} \times \bar{M}_{2}$ (cf. [4]). Moreover it is easily checked that Riemannian product metric $\bar{g}$ on $\bar{M}_{1} \times \bar{M}_{2}$ is a non-Kaehler Hermitian metric on the complex manifold.

Let $M^{2 n}$ be a complex submanifold of codimension $2 p$ of $\bar{M}_{1} \times \bar{M}_{2}$ with the Hermitian structure which is defined as above, and $i: M \rightarrow \bar{M}_{1} \times \bar{M}_{2}$ be the immersion.

EXAMPLE. Let $P_{n}^{2}(\boldsymbol{C})(i=1,2)$ be complex projective spaces with homogeneous coordinates $\left(z_{0}^{2}, \cdots, z_{n}^{2}\right)$ and constant holomorphic sectional curvature 4 . Let $M$ be a complex hypersurface of $P_{n}^{1}(\boldsymbol{C}) \times P_{n}^{2}(\boldsymbol{C})$ defined by $\sum_{j=0}^{n} z_{j}^{1} z_{j}^{2}=0$ and $\left(M, T^{2}\right)$ be the torus bundle over $M$ such that the following diagram is commutative;

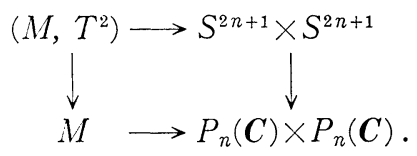

Then $\left(M, T^{2}\right)$ is the complex hypersurfaces of $S^{2 n+1} \times S^{2 n+1}$. Moreover, $M$ is diffeomorphic to $U(n+1) / U(n-1) \times T^{2}$ (Kaehler $C$-space) and $\left(M, T^{2}\right)$ is diffeomorphic to $U(n+1) / U(n-1)$ (Complex Stiefel manifold).

We take orthonormal normal vectors $N_{1}, \cdots, N_{2 p}$ to $M$ in such a way that $N_{2 q}=J N_{2 q-1}(q=1, \cdots, p)$. Suppose that the vector fields $\bar{\xi}_{\imath}(i=1,2)$ are not always tangent to $M$. Then there exists such a point $x \in M$ that the normal parts of $\bar{\xi}_{2}(i=1,2)$ do not vanish, because $\bar{\xi}_{2}=J \bar{\xi}_{1}$. At this point, we can choose the unit normal frame to $M$ in such a way that, $N_{\imath}(i=1,2)$ are the normal 
directions of $\bar{\xi}_{\imath}(i=1,2)$ and extend them to local fields. Hence $\bar{\xi}_{\iota}$ can be written as a sum of the tangential components and the normal components in the following way,

$$
\bar{\xi}_{\jmath}=i_{*} \xi_{\jmath}+r N, \quad(\jmath=1,2) .
$$

Then $\xi$, and $r$ define vector fields and a function on $M$ respectively. Let $X$ be a tangent vector field on $M$. Then we immediately get

$$
\begin{aligned}
& \bar{\eta}_{j}\left(i_{*} X\right)=\bar{g}\left(\bar{\xi}_{j}, i_{*} X\right)=g\left(\hat{\xi}_{\jmath}, X\right), \\
& \bar{\eta}_{j}\left(N_{j}\right)=\bar{g}\left(\bar{\xi}_{j}, N_{j}\right)=r \quad(j=1,2, \quad 0 \leqq r \leqq 1), \\
& \bar{\eta}_{j}\left(N_{k}\right)=0 \quad(\jmath=1,2, \quad k=1, \cdots, 2 p, \quad \jmath \neq k),
\end{aligned}
$$

where $g$ is the induced Riemannian metric on $M$. The transforms $\bar{\phi}_{j} l_{*} X$ and $\bar{\phi}_{j} N_{\alpha}(\jmath=1,2, \alpha=1,2, \cdots, 2 p)$ of $X$ and $N_{\alpha}$ by $\bar{\phi}_{\jmath}$ can be written as

$$
\begin{aligned}
& \bar{\phi}_{1} i_{*} X=i_{*} \phi_{1} X+\sum_{\alpha=1}^{2 p} v^{\alpha}(X) N_{\alpha} \\
& \bar{\phi}_{2} \imath_{*} X=i_{*} \phi_{2} X+\sum_{\alpha=1}^{2 p} w^{\alpha}(X) N_{\alpha} \\
& \bar{\phi}_{1} N_{\alpha}=-i_{*} V_{\alpha}+\sum_{\beta=1}^{2 p} \mu_{\alpha \beta} N_{\beta}, \\
& \bar{\phi}_{2} N_{\alpha}=-i_{*} W_{\alpha}+\sum_{\beta=1}^{2 p} \nu_{\alpha \beta} N_{\beta},
\end{aligned}
$$

where $\phi$,'s define skew-symmetric linear transformations of the tangent bundle of $M$, while $v^{\alpha}, w^{\alpha}, V_{\alpha}, W_{\alpha}, \mu_{\alpha \beta}$ and $\nu_{\alpha \beta}$ define 1-forms, vector fields and functions on a neighborhood of a point of $M$ respectively. We easily see that $\mu_{\alpha_{\beta}}$ and $\nu_{\alpha \beta}$ are skew-symmetric with respect to $\alpha$ and $\beta$ and that

$$
g\left(V_{\alpha}, X\right)=v^{\alpha}(X), \quad g\left(W_{\alpha}, X\right)=w^{\alpha}(X) \quad(\alpha=1, \cdots, 2 p) .
$$

Since $J N_{1}=N_{2}$ and $J N_{2}=-N_{1}$ hold, using (2.1), (2.3) and (2.6), we have

$$
V_{1}+W_{1}-r \xi_{2}=0, \quad V_{2}+W_{2}+r \xi_{1}=0,
$$

$$
\mu_{1,2}+\nu_{1,2}+r^{2}=1
$$

Similarly, from $J N_{2 q-1}=N_{2 q}$ and $J N_{2 q}=-N_{2 q-1}(q=2,3, \cdots, p)$, we have

$$
V_{2 \alpha-1}+W_{2 \alpha-1}=V_{2 \alpha}+W_{2 \alpha}=0,
$$

$$
\begin{aligned}
& \mu_{2 \alpha-1,2 \alpha}+\nu_{2 \alpha-1,2 \alpha}=1, \\
& \mu_{2 \alpha-1, \gamma}+\nu_{2 \alpha-1, \gamma}=\mu_{2 \beta, \gamma}+\nu_{2, \beta, \gamma}=0 \quad(\gamma \neq 2 \alpha, 2 \beta-1) .
\end{aligned}
$$

Let $\bar{P}$ be the almost product structure defined in $\S 1$. Since $\bar{P}_{1} \overline{\bar{\xi}}_{1}=\bar{\xi}_{1}^{\prime}$ and $\bar{P}_{2} \bar{\xi}_{2}=\bar{\xi}_{2}^{\prime}$ holds, $\bar{P} \bar{\xi}_{1}=\bar{\xi}_{1}$ and $\bar{P} \bar{\xi}_{2}=-\bar{\xi}_{2}$. From this, using (1.1), (1.2) and (2.2), 
we get

$$
\begin{aligned}
& P \xi_{1}=\xi_{1}-r U_{1}, \quad P \xi_{2}=-\xi_{2}-r U_{2}, \\
& u^{1}\left(\xi_{1}\right)=r\left(1-\lambda_{1,1}\right), \quad u^{2}\left(\xi_{2}\right)=-r\left(1+\lambda_{2,2}\right), \\
& u^{\alpha}\left(\xi_{1}\right)=-r \lambda_{1, \alpha}, \quad u^{\beta}\left(\xi_{2}\right)=-r \lambda_{2, \beta} \quad(\alpha \neq 1, \beta \neq 2) .
\end{aligned}
$$

From the definition of $\bar{P}$, we have

$$
\bar{P} \bar{\phi}_{1}=\bar{\phi}_{1}=\bar{\phi}_{1} \bar{P}, \quad \bar{P} \bar{\phi}_{2}=-\bar{\phi}_{2}=\bar{\phi}_{2} \bar{P} .
$$

Applying $\bar{P}$ to $J N_{1}=N_{2}$ and $J N_{2}=-N_{1}$, using (2.1), we have

$$
\begin{aligned}
& U_{1}=V_{2}-W_{2}+r \xi_{1}, \quad U_{2}=-V_{1}+W_{1}-r \xi_{2}, \\
& \lambda_{1,1}=-\mu_{2,1}+\nu_{2,1}+r^{2}, \quad \lambda_{2,2}=\mu_{1,2}-\nu_{1,2}-r^{2}, \\
& \lambda_{1, \alpha}=-\mu_{2, \alpha}+\nu_{2, \alpha}, \quad \lambda_{2, \beta}=\mu_{1, \beta}-\nu_{1, \beta}, \quad(\alpha \neq 1, \quad \beta \neq 2),
\end{aligned}
$$

because of (2.14). From these equations and (2.7) (2.9), we obtain

$$
\begin{aligned}
& U_{2}=-2 V_{1}, \quad U_{1}=-2 W_{2}, \\
& \lambda_{2,2}=2 \mu_{1,2}-1, \quad \lambda_{1,1}=2 \nu_{2,1}+1, \\
& \lambda_{2,1}=0, \quad \lambda_{2, \alpha}=2 \mu_{1, \alpha} \quad(\alpha \neq 2), \\
& \lambda_{1,2}=0, \quad \lambda_{1, \alpha}=2 \nu_{2, \alpha} \quad(\alpha \neq 1) .
\end{aligned}
$$

In the same way, we have, for $N_{k}(k=3,4, \cdots, 2 p)$

$$
\begin{aligned}
& U_{2 q}=-V_{2 q-1}+W_{2 q-1}, \quad U_{2 q-1}=V_{2 q}-W_{2 q} \quad(q=2,3, \cdots, p), \\
& \lambda_{2 q, \beta}=\mu_{2 q-1, \beta}-\nu_{2 q-1, \beta}, \quad \lambda_{2 q-1, \beta}=-\mu_{2 q, \beta}+\nu_{2 q, \beta} \quad(\beta=1,2, \cdots, 2 p) .
\end{aligned}
$$

These equations, (2.10) and (2.11) imply that

$$
\begin{aligned}
& U_{2 q}=-2 V_{2 q-1}, \quad U_{2 q-1}=-2 W_{2 q}, \\
& \lambda_{2 q, 2 q}=2 \mu_{2 q-1,2 q}-1, \quad \lambda_{2 q-1,2 q-1}=2 \nu_{2 q, 2 q-1}+1, \\
& \lambda_{2 q, \beta}=2 \mu_{2 q-1, \beta}, \quad \lambda_{2 q-1, \gamma}=2 \nu_{2 q, \gamma}, \\
& \lambda_{2 q, 2 q-1}=0 \quad(\beta \neq 2 q, \gamma \neq 2 q-1) .
\end{aligned}
$$

On the other hand, since $\bar{\phi}_{j} \bar{\xi}_{k}=0(j=1,2, k=1,2)$ hold, using (2.2), (2.5) and (2.6), we have

$$
\begin{aligned}
& \phi_{1} \xi_{j}=r V_{\jmath}, \quad \phi_{2} \xi_{j}=r W_{\jmath}, \\
& v^{\alpha}\left(\xi_{j}\right)=r \mu_{\alpha, \jmath}, \quad w^{\alpha}\left(\xi_{j}\right)=r \nu_{\alpha, \jmath} \quad(\alpha=1,2, \cdots, 2 p) .
\end{aligned}
$$

Since $\bar{M}_{1}$ and $\bar{M}_{0}$ are Sasakian manifolds, one obtains that 


$$
\bar{\nabla}_{X} \bar{\xi}_{1}=\bar{\phi}_{1} X, \quad \bar{\nabla}_{X} \bar{\xi}_{2}=\bar{\phi}_{2} X
$$

Then, using Gauss, Weingarten equations and (2.5), we have

$$
\begin{aligned}
& \nabla_{X} \xi_{1}=\phi_{1} X+r A_{1} X, \quad \nabla_{X} \xi_{2}=\phi_{2} X+r A_{2} X, \\
& \operatorname{grad} r=V_{1}-A_{1} \xi_{1}=W_{2}-A_{2} \xi_{2}, \\
& V_{\alpha}=A_{\alpha} \xi_{1}+r S_{1, \alpha} \quad(\alpha \neq 1), \\
& W_{\alpha}=A_{\alpha} \xi_{2}+r S_{2, \alpha} \quad(\alpha \neq 2),
\end{aligned}
$$

where $S_{\alpha \beta}$ are dual vectors of 1 -forms $s_{\alpha \beta}(X)$. Hence

$$
\operatorname{div} \xi_{1}=r \operatorname{trace} A_{1}, \quad \operatorname{div} \xi_{2}=r \operatorname{trace} A_{2} .
$$

Here, for simplicity, we have written $A_{\alpha}$ instead of $A_{N_{\alpha}}$ for a frame $N_{1}, N_{2}$, $\cdots, N_{2 p}$ for $N_{x} M$. In the same way, for any $X \in T(M)$, we have

$$
\left(\bar{\nabla}_{X} \bar{\phi}_{j}\right) N_{\alpha}=\bar{\eta}_{\jmath}\left(N_{\alpha}\right) \bar{P}_{j} X-\bar{g}\left(\bar{P}_{j} X, N_{\alpha}\right) \bar{\xi}_{j} \quad(j=1,2, \alpha=1,2, \cdots, 2 p) .
$$

Since $\bar{P}_{1}=(I+P) / 2$ and $\bar{P}_{2}=(I-P) / 2$, making use of Gauss, Weingarten equations and $(2.2) \sim(2.6)$, we get

$$
\begin{aligned}
& \nabla_{X} V_{1}=-(r / 2)(X+P X)+(1 / 2) u^{1}(X) \xi_{1}+\phi_{1} A_{1} X+\sum_{\alpha=1}^{2 p}\left(s_{1, \alpha}(X) V_{\alpha}-\mu_{1, \alpha} A_{\alpha} X\right), \\
& \nabla_{X} V_{\alpha}=(1 / 2) u^{\alpha}(X) \xi_{1}+\phi_{1} A_{\alpha} X+\sum_{\alpha=1}^{2 p}\left(s_{\alpha \beta}(X) V_{\beta}-\mu_{\alpha \beta} A_{\beta} X\right) \quad(\alpha \neq 1) . \\
& \nabla_{X} W_{2}=-(r / 2)(X-P X)-(1 / 2) u^{2}(X) \xi_{2}+\phi_{2} A_{2} X+\sum_{\alpha=1}^{2 p}\left(s_{2, \alpha}(X) W_{\alpha}-\nu_{2, \alpha} A_{\alpha} X\right), \\
& \nabla_{X} W_{\alpha}=-(1 / 2) u^{\alpha}(X) \xi_{2}+\phi_{2} A_{\alpha} X+\sum_{\alpha=1}^{2 p}\left(s_{\alpha \beta}(X) W_{\beta}-\nu_{\alpha \beta} A_{\beta} X\right) \quad(\alpha \neq 2) .
\end{aligned}
$$

From above, using (2.12) and (2.13), we obtain

$$
\begin{aligned}
\operatorname{div} V_{1}= & -r n-(r / 2) \operatorname{trace} P+(r / 2)\left(1-\lambda_{11}\right) \\
& +\sum_{\alpha=1}^{2 p}\left(s_{1, \alpha}\left(V_{\alpha}\right)-\mu_{1, \alpha} \operatorname{trace} A_{\alpha}\right), \\
\operatorname{div} V_{\alpha}= & -(r / 2) \lambda_{1, \alpha}+\sum_{\beta=1}^{2 p}\left(s_{\alpha \beta}\left(V_{\beta}\right)-\mu_{\alpha \beta} \operatorname{trace} A_{\beta}\right) \quad(\alpha \neq 1) \\
\operatorname{div} W_{2}= & -r n+(r / 2) \operatorname{trace} P+(r / 2)\left(1+\lambda_{2,2}\right) \\
& +\sum_{\alpha=1}^{2 p}\left(s_{2, \alpha}\left(W_{\alpha}\right)-\nu_{2, \alpha} \operatorname{trace} A_{\alpha}\right), \\
\operatorname{div} W_{\alpha}= & (r / 2) \lambda_{2, \alpha}+\sum_{\beta=1}^{2 p}\left(s_{\alpha \beta}\left(W_{\beta}\right)-\nu_{\alpha \beta} \operatorname{trace} A_{\beta}\right) \quad(\alpha \neq 2) .
\end{aligned}
$$

Now we prove the following,

THEOREM. Let $\bar{M}_{1} \times \bar{M}_{2}$ be the Riemannian product of Sasakıan manifolds, and $M$ be its compact complex submanifold with respect to the complex structure that is defined by (2.1). Then 
(1) $\bar{\xi}_{\imath}(i=1,2)$ are tangent to $M$,

(2) $\quad M$ is a minimal submanifold of $\bar{M}_{1} \times \bar{M}_{2}$.

Proof. First of all, we calculate $\operatorname{div} r \xi_{2}$ and $\operatorname{div} r \xi_{2}$. Making use of (2.17) $\sim$ (2.20), we get

$$
\begin{aligned}
\operatorname{div} r \xi_{2} & =\left(\xi_{2} r\right)+r^{2} \operatorname{trace} A_{2} \\
& =g\left(\operatorname{grad} r, \xi_{2}\right)+r^{2} \operatorname{trace} A_{2} \\
& =g\left(V_{1}-A_{1} \xi_{1}, \xi_{2}\right)+r^{2} \operatorname{trace} A_{2} \\
& =r \mu_{1,2}-g\left(A_{1} \xi_{2}, \xi_{1}\right)+r^{2} \operatorname{trace} A_{2} \\
& =r \mu_{1,2}-g\left(W_{1}-r S_{2,1}, \xi_{1}\right)+r^{2} \operatorname{trace} A_{2} \\
& =r \mu_{1,2}+s_{2,1}\left(r \xi_{1}\right)+r^{2} \operatorname{trace} A_{2} .
\end{aligned}
$$

In the same way, we have

$$
\operatorname{div} r \xi_{1}=r \nu_{2,1}+s_{1,2}\left(r \xi_{2}\right)+r^{2} \text { trace } A_{1} .
$$

Hence, making use of (2.7), (2.10), (2.15), (2.16), (2.21) and (2.22), we have

$$
\begin{aligned}
0= & \operatorname{div} V_{1}+\operatorname{div} W_{1}-\operatorname{div} r \xi_{2} \\
= & -r n-(r / 2) \operatorname{trace} P+(r / 2)\left(1-\lambda_{1,1}\right) \\
& +\sum_{\alpha=1}^{2 p}\left(s_{1, \alpha}\left(V_{\alpha}\right)-\mu_{1, \alpha} \operatorname{trace} A_{\alpha}\right) \\
& +(r / 2) \lambda_{2,1}+\sum_{\alpha=1}^{2 p}\left(s_{1, \alpha}\left(W_{\alpha}\right)-\nu_{1, \alpha} \operatorname{trace} A_{\alpha}\right) \\
& -r \mu_{1,2}-s_{2,1}\left(r \xi_{1}\right)-r^{2} \operatorname{trace} A_{2} \\
= & -r n-(r / 2) \operatorname{trace} P-(r / 2)\left(\lambda_{1,1}+\lambda_{2,2}\right)-\operatorname{trace} A_{2} .
\end{aligned}
$$

Consequently, we get

$$
\text { trace } A_{2}=-r\left(n+(\operatorname{trace} P) / 2+\left(\lambda_{1,1}+\lambda_{2,2}\right) / 2\right) \text {. }
$$

Similarly, we have

$$
\begin{aligned}
0= & \operatorname{div} V_{2}+\operatorname{div} W_{2}+\operatorname{div} r \xi_{1} \\
= & -(r / 2) \lambda_{1,2}+\sum_{\alpha=1}^{2 p}\left(s_{2, \alpha}\left(V_{\alpha}\right)-\mu_{2, \alpha} \operatorname{trace} A_{\alpha}\right) \\
& -r n+(r / 2) \operatorname{trace} P+r\left(1+\lambda_{2,2}\right) / 2 \\
& +\sum_{\alpha=1}^{2 p}\left(s_{2, \alpha}\left(W_{\alpha}\right)-\nu_{2, \alpha} \operatorname{trace} A_{\alpha}\right) \\
& +r \nu_{2,1}+s_{1,2}\left(r \xi_{2}\right)+r^{2} \operatorname{trace} A_{1} \\
= & \operatorname{trace} A_{1}-r n+(r / 2) \operatorname{trace} P+r\left(\lambda_{1,1}+\lambda_{2,2}\right) / 2 .
\end{aligned}
$$


Hence we obtain

$$
\text { trace } A_{1}=r\left(n-(1 / 2) \text { trace } P-\left(\lambda_{1,1}+\lambda_{2,2}\right) / 2\right) \text {. }
$$

By quite the same computation, using (2.10) and (2.11), we have

$$
\begin{aligned}
0= & \operatorname{div} V_{2 q-1}+\operatorname{div} W_{2 q-1} \\
= & -(r / 2) \lambda_{1,2 q-1}+\sum_{a=1}^{2 p}\left(s_{2 q-1, \alpha}\left(V_{\alpha}\right)-\mu_{2 q-1, \alpha} \operatorname{trace} A_{\alpha}\right) \\
& +(r / 2) \lambda_{2,2 q-1}+\sum_{a=1}^{2 p}\left(s_{2 q-1, \alpha}\left(W_{\alpha}\right)-\nu_{2 q-1, \alpha} \operatorname{trace} A_{\alpha}\right) \\
= & (r / 2)\left(\lambda_{2,2 q-1}-\lambda_{1,2 q-1}\right)-\operatorname{trace} A_{2 q}-r\left(s_{2 q-1,2}\left(\xi_{1}\right)-s_{2 q-1,1}\left(\xi_{2}\right)\right),
\end{aligned}
$$

and

$$
\begin{aligned}
0 & =\operatorname{div} V_{2 q}+\operatorname{div} W_{2 q} \\
& =(r / 2)\left(\lambda_{2,2 q}-\lambda_{1,2 q}\right)+\operatorname{trace} A_{2 q-1}-r\left(s_{2 q, 2}\left(\xi_{1}\right)-s_{2 q, 1}\left(\xi_{2}\right)\right) .
\end{aligned}
$$

Consequently, we get

$$
\begin{aligned}
& \operatorname{trace} A_{2 q-1}=r\left(\left(\lambda_{1,2 q}-\lambda_{2,2 q}\right) / 2-s_{2 q, 1}\left(\xi_{2}\right)+s_{2 q, 2}\left(\xi_{1}\right)\right), \\
& \operatorname{trace} A_{2 q}=r\left(\left(\lambda_{2,2 q-1}-\lambda_{1,2 q-1}\right) / 2-s_{2 q-1,2}\left(\xi_{1}\right)+s_{2 q-1,1}\left(\xi_{2}\right)\right) \\
& \quad(q=2,3, \cdots, 2 p) .
\end{aligned}
$$

Then from (2.20), (2.23) and (2.24), we obtain

$$
\operatorname{div} \xi_{1}-\operatorname{div} \xi_{2}=r\left(\operatorname{trace} A_{1}-\operatorname{trace} A_{2}\right)=2 r^{2} n .
$$

Since $M$ is compact, by Green's theorem, we have

$$
0=\int_{M}\left(\operatorname{div} \xi_{1}-\operatorname{div} \xi_{2}\right) * 1=2 n \int_{M} r^{2 * 1}
$$

where $* 1$ is a volume element on $M$. From this, $r^{2}=0$ on $M$, that is $\xi_{j} \in T_{x} M$ $(j=1,2)$. Hence by $(2.23) \sim(2.26)$, trace $A_{a}=0 \quad(\alpha=1,2, \cdots, 2 p)$. Consequently, $M$ is a minimal submanifold.

\section{REFERENCES}

[1] BlaiR, D., Contact manifolds in Riemannian geometry, Lecture Notes in Math., vol. 509, Springer-Verlag.

[2] Calabi, E. AND B. Eckmans, A class of compact complex manifolds which are not algebraic, Ann. of Math., 58 (1953), 494-500.

[3] Ludden, G. D. And M. OKumura, Some integral formulas and their applications to hypersurfaces of $S^{n} \times S^{n}$, J. Differential Geom., 9 (1974), 617-631.

[4] Morimoto, A., On normal almost contact structures, J. Math. Soc. Japan, 15 (1963), 420-436. 
[5] OGiue, K., Differential geometry of Kaehler submanifolds, Advances in Math., 13 (1974), 73-114.

[6] OKumura, M., Submanifolds of a Kaehlerian manifold and a Sasakian manifold, Lecture Notes in Michigan State Univ. 1971.

Department of Mathematics

SAITAMA University 